\title{
Utilización de plantas medicinales en cuatro localidades de la zona sur de Manabí, Ecuador
}

\author{
Use of medicinal plants in four localities of southern \\ Manabí, Ecuador
}

\author{
Alfredo Jiménez González ${ }^{1}$, Karla Julexi Mora Zamora², \\ Sonia Rosete Blandariz ${ }^{3}$, César Alberto Cabrera Verdesoto ${ }^{4}$
}

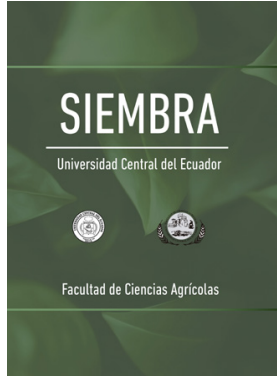

\footnotetext{
${ }^{1}$ Universidad Estatal del Sur de Manabí, Facultad de Ciencias Naturales y de la Agricultura, Carrera de Ingeniería Forestal. Centro de Estudios de Biotecnología (CEB). Campus Los Ángeles. Km 1 1⁄2 Vía A Noboa. Código postal 130650. Jipijapa, Manabí, Ecuador.

凶alfredo.jimenez@unesum.edu.ec

2. https://orcid.org/0000-0002-1768-5566

${ }^{2}$ Universidad Estatal del Sur de Manabí, Facultad de Ciencias Naturales y de la Agricultura, Carrera de Ingeniería Forestal. Campus Los Ángeles. Km 1 1/2 Vía A Noboa. Código postal 130650. Jipijapa, Manabí, Ecuador.

凶karla.mora@unesum.edu.ec

D. https://orcid.org/0000-0001-6636-4454

${ }^{3}$ Universidad Estatal del Sur de Manabí, Facultad de Ciencias Económicas, Carrera de Turismo. Campus Los Ángeles. Km 1 1 1/2 Vía A Noboa. Código postal 130650. Jipijapa, Manabí, Ecuador.

凶sonia.rosete@unesum.edu.ec

Q https://orcid.org/0000-0002-8596-5121

${ }^{4}$ Universidad Estatal del Sur de Manabí, Facultad de Ciencias Naturales y de la Agricultura, Carrera de Ingeniería Forestal. Campus Los Ángeles. Km 1 1/2 Vía A Noboa. Código postal 130650. Jipijapa, Manabí/Ecuador.

$\bowtie$ cesar.cabrera@unesum.edu.ec

Q h http://orcid.org/0000-0001-5101-3520

*Autor de correspondencia:

alfredo.jimenez@unesum.edu.ec
}

\section{SIEMBRA}

https://revistadigital.uce.edu.ec/index.php/SIEMBRA ISSN-e: $2477-8850$

ISSN: $1390-8928$

Periodicidad: semestral

vol. 8, núm. 2, 2021

siembra.fag@uce.edu.ec

DOI: https://doi.org/10.29166/siembra.v8i2.3223

Esta obra está bajo una licencia internacional Creative Commons Atribución-NoComercial

\section{Resumen}

Los saberes que posee la población manabita sobre la utilización de plantas medicinales contribuyen a cubrir vacíos de información sobre la medicina natural y tradicional en Ecuador. Esta investigación de alcance descriptivo tuvo el objetivo de profundizar en el conocimiento de los usos medicinales de plantas en Membrillal, Julcuy, Pedro Pablo Gómez y Quimis, en el sur de Manabi. Los datos fueron facilitados por 200 entrevistados, durante el periodo comprendido entre julio y noviembre de 2019. Además del componente sociocultural (edad, género, nivel educacional), la entrevista indagó sobre las plantas, las partes que se usan, las formas de uso, la abundancia, el ambiente donde crecen, la frecuencia de uso, la distancia desde sus casas al lugar de recolección, finalidades de usos y época de recolección. También se estudió las características fitoquímicas de las plantas, que validan los usos medicinales. En total fueron citadas 144 especies vegetales con algún uso medicinal, pertenecientes a 58 familias botánicas y 118 géneros. Las familias más citadas fueron Fabaceae, Lamiaceae, Asteraceae y Rutaceae. Las especies Mentha spicata L. y Cymbopogon citratus (DC.) Stapf, alcanzaron el mayor número de citas. Las partes de las plantas más utilizadas fueron las hojas $(65,5 \%)$, para hacer infusiones $(70,5 \%)$. Las enfermedades del sistema digestivo, infecciosas y parasitarias fueron las enfermedades tratadas con las plantas medicinales en Membrillal, Julcuy, Pedro Pablo Gómez y Quimis, con una frecuencia de 35,9\%, 27,8 \%, 30,3\% y 35,0 \% de los entrevistados, respectivamente. Los principios bioactivos que están contenidos en las especies de plantas medicinales fueron los flavonoides, fenoles, triterpenos y alcaloides.

Palabras clave: conocimiento, etnobotánica, principios bioactivos, usos.

\begin{abstract}
Knowledge of Manabians about medicinal plants use contributes to filling gaps of information on natural and traditional medicine in Ecuador. This descriptive research had the objective of deepening the uses knowledge of medicinal plants in Membrillal, Julcuy, Pedro Pablo Gómez and Quimis, Southern Manabi. The data were provided by 200 interviewees from July to November 2019. In addition to the sociocultural interview component
\end{abstract}


(age, gender, educational level), the interview also included information about knowledge of the medicinal plants, plant parts, use, the forms of use, frequency of use, purposes of use, the abundance in the region, the environment surrounding the plant, distance from home to plant collection, and time of collection. The phytochemical characteristics of the plant was also studied, which validated the medicinal use. In total 144 plant species with some medicinal use were identified by the interviewees. The plant species identified belonged to 58 botanical families and 118 genus. The most frequently identified families were Fabaceae, Lamiaceae, Asteraceae and Rutaceae. The species Mentha spicata L. and Cymbopogon citratus (DC.) Stapf, were the most frequently described. The plant parts most frequently used were leaves (65.5\% of interviewees) to make infusions ( $70.5 \%$ of interviewees). Digestive system diseases as well as infectious and parasitic diseases were the mostly treated diseases with the medicinal plants, with a interviewee frequency of $35.9 \%, 27.8 \%, 30.3 \%$ and $35.0 \%$ in Membrillal, Julcuy, Pedro Pablo Gomez and Quimis, respectively. The bioactive principles of the medicinal plants described by the interviewees were flavonoids, phenols, triterpenoids and alkaloids.

Keywords: knowledge, ethnobotany, bioactive principles, uses.

\section{Introducción}

La sociedad científica muestra una creciente preocupación por las plantas medicinales y la conservación de los recursos naturales (Rodríguez Guerra et al., 2019). El uso terapéutico de plantas medicinales, como sustitutas de las medicinas farmacéuticas, se aplica desde la antigüedad para curar o aliviar las enfermedades. La población en las comunidades rurales manifiesta que la primera alternativa que tiene para atender su problema de salud es el tratamiento con plantas medicinales (Gallegos-Zurita, 2016).

Del total de especies vegetales que habitan el planeta, solo un porcentaje menor al $10 \%$ han sido evaluadas científicamente con fines medicinales o terapéuticos, por lo que un estimado indica que 15.000 plantas medicinales se encuentran en peligro de extinción (Organización Panamericana de la Salud [OPS], 2019). En este mismo sentido, se calcula que al menos 1.000 millones de personas, sin incluir las que se encuentran en América del Norte y Europa, utilizan hierbas medicinales para el tratamiento de enfermedades gastrointestinales. Las plantas silvestres recolectadas en los bosques y zonas cercanas proporcionan materias primas importantes (Organización de las Naciones Unidas para la Alimentación y la Agricultura [FAO] \& Programa de las Naciones Unidas para el Medio Ambiente [PNUMA], 2020).

A decir de Bermúdez et al. (2005), los países en desarrollo han perdido un importante conocimiento tradicional respecto al uso de las plantas medicinales, por lo que la investigación etnobotánica puede ayudar a recuperar el conocimiento ancestral y proteger de manera simultánea la biodiversidad.

Por todo lo anteriormente expuesto y con el objetivo de profundizar en el conocimiento sobre la utilización de las plantas de interés medicinal en las localidades Membrillal, Julcuy, Pedro Pablo Gómez y Quimis, Manabí, Ecuador, se realizó la presente investigación en el marco del proyecto "Componentes de la diversidad biológica empleados por las familias manabitas en la medicina natural y tradicional”, ejecutado por las carreras de Ingeniería Forestal y de Turismo, de la Universidad Estatal del Sur de Manabí, lo cual justifica la problemática sobre la pérdida del conocimiento ancestral por las familias manabitas, en el marco del programa Ecoturístico-Forestal de dicha institución.

\section{Materiales y métodos}

La presente investigación es de tipo descriptiva, puesto que recoge información independiente sobre la utilización de plantas medicinales en cuatro localidades de la zona Sur de Manabí, a saber: Membrillal, Julcuy, Pedro Pablo Gómez y Quimis; en este mismo sentido se emplearon los siguientes métodos de investigación:

- Observación: las visitas de campo realizadas permitieron determinar la situación global de la comunidad, en los ámbitos socio-culturales, económicos, ambientales y políticos, según los criterios de Reyes Vargas et al. (2019). Desde el inicio se contó con el apoyo de los líderes locales con los cuales se realizaron conversatorios, para dar a conocer el propósito de la investigación, a fin de que lo aprobaran y comunicaran a las familias sobre la intervención que se realizaría.

- Deductivo: consistió en extraer una conclusión con base en una premisa o una serie de proposiciones que se asumen como verdaderas. Lo anterior quiere decir que se está usando la lógica para obtener un resul- 
tado, solo con base en un conjunto de afirmaciones que se dan por ciertas, en este caso, las propiedades medicinales que le atribuyen las personas entrevistadas a las plantas.

- Descriptivo: en lo que respecta al conocimiento de los entrevistados en las localidades en estudio sobre los usos medicinales de las plantas. En este aspecto, para hacer la recopilación de información etnobotánica sobre la utilización de las plantas de interés medicinal en las localidades objeto de estudio, se realizaron 200 entrevistas (50 en cada localidad), distribuidas en 38 núcleos familiares, vistos en esta investigación como familias, durante los meses de julio y noviembre de 2019, mediante varias expediciones (entre cuatro a cinco visitas/localidad) efectuadas a dichas localidades.

Las entrevistas contaron con preguntas relacionadas con la edad, el género, el nivel educacional, lo que permitió obtener datos sobre las plantas que utilizan como medicinales, también sobre las partes del vegetal usadas, las formas de empleo (cocido, infusión, crudo, emplasto, sahumerio, extracción de aceites y baños), la abundancia, el ambiente donde se desarrollan, la frecuencia de uso, la distancia a la que se encuentran dichas plantas de sus hogares, la finalidad de usos y la época de recolección, también se presentan las características fitoquímicas de las plantas más citadas por los entrevistados, con la finalidad de validar los usos medicinales de esas plantas por los pobladores de las localidades involucradas en esta investigación.

\subsection{Tamaño de la muestra}

La selección de la muestra para realizar las entrevistas no estuvo establecida bajo ningún criterio de edad, sexo u ocupación, dado que el método a utilizar para esta entrevista se basa en seleccionar a la persona con mayor conocimiento, según lo describe la literatura; así, el muestreo de bola de nieve es un tipo de muestreo no probabilístico que se utiliza cuando los participantes potenciales son difíciles de encontrar o si la muestra está limitada a un subgrupo muy pequeño de la población. El método de muestreo de bola de nieve se basa en referencias de sujetos iniciales para generar sujetos adicionales, de ahí deriva su nombre "bola de nieve" (Jiménez González et al., 2021; López-Roldán y Fachelli, 2015). Para el establecimiento de la población y la muestra, se tomó en cuenta el cálculo del tamaño de la muestra conociendo el tamaño de la población (Torres et al., 2006), según la ecuación [1].

$$
n=\frac{\mathrm{N} * Z^{2} * \mathrm{p} * \mathrm{q}}{d^{2} *(N-1)+Z^{2} * p * q}
$$

donde:

- $\quad N=$ tamaño de la población;

- $\quad \mathrm{Z}=$ nivel de confianza;

- $\quad p=$ probabilidad de éxito, o proporción esperada;

- $q=$ probabilidad de fracaso;

- $\quad d=$ precisión (error máximo admisible en términos de proporción).

Las enfermedades o afecciones tratadas con la etnomedicina, mencionadas por los entrevistados, se agruparon de acuerdo con la Clasificación Estadística Internacional de Enfermedades y Problemas Relacionados con la Salud de la Organización Mundial de la Salud (OMS, 2018).

\subsection{Caracterización de las plantas de interés medicinal}

En la caracterización desde el punto de vista fitoquímico de las plantas de interés medicinal para las localidades objeto de estudio se verificaron las estructuras más empleadas, a saber, las partes aéreas de las plantas (flores, 
hojas, semillas, tallos), sobre la base de una revisión documental. (Aguirre, 2012; Jiménez-González et al., 2010; Jiménez González et al., 2017; Jiménez et al., 2021). Para analizar el manejo de las especies medicinales cuyos usos están en las raíces se buscó información documental (Manzanero-Medina et al., 2009). En el caso de la indagación sobre los usos medicinales tradicionales de las plantas se buscó información bibliográfica sobre la fitoquímica (Carvajal Rojas et al., 2009; Gil-Rodríguez et al., 2020; Olascuaga-Castillo et al., 2020; Pérez-Chauca et al., 2020; Ramírez Cárdenas et al., 2017).

Para determinar la percepción de la abundancia de plantas utilizadas en medicina tradicional se establecieron categorías del 1 al 5, donde: 5 es muy alto; 4 es alto; 3 es medianamente alto; 2 es bajo y 1 es muy bajo.

La nomenclatura de las especies de flora citadas se determinó mediante la revisión de la base Trópicos, del Sistema de Información Botánica del Jardín Botánico de Missouri (Tropicos.org, 2020) y en el Catálogo de la Vida (Roskov et al., 2019). En lo referente a los nombres comunes, éstos fueron proporcionados por los guías locales (Jiménez González et al., 2016). En este aspecto se concuerda con Kvist et al. (2001), quienes concluyeron que cada método contribuye de manera significativa a varios, pero nunca a todos los objetivos y que siempre hay factores que favorecen o dificultan los métodos, debido tanto a los recursos y medios disponibles para el estudio como al entorno en que éste se realiza.

Por lo antes expuesto en la determinación de la relación entre nombres vernáculos y nombres científicos se tuvo en cuenta lo planteado por Kvist et al. (2001), quienes aseguran que, los informantes refieren a las plantas medicinales con sus nombres locales (vernaculares). Muchas veces brindan información sobre plantas difíciles o imposibles de encontrar y recolectar para su identificación científica. Si no se conocen los nombres locales correspondientes a las especies científicas, aquella información es poco útil para los investigadores. De acuerdo con esos autores, la relación entre los nombres locales y los nombres científicos también es imprescindible para entender el sistema de clasificación de las plantas usadas por las poblaciones locales.

\subsection{Análisis estadístico}

Los datos fueron analizados según las tablas de frecuencia en el software SPSS Vers. 22 para Windows.

\section{Resultados}

En total fueron citadas 144 especies de plantas con al menos un uso medicinal reconocido, distribuidas en 58 familias botánicas y 118 géneros; las familias Fabaceae, Lamiaceae, Asteraceae y Rutaceae fueron las más citadas (Tabla 1). De acuerdo con los resultados relacionados con la percepción de la abundancia de plantas utilizadas en medicina tradicional, el $36 \%$ de los pobladores indicaron que es muy alta, mientras que el 5,33\% indicaron que la percepción es muy baja.

Tabla 1. Lista de especies de interés medicinal para los habitantes de Membrillal, Julcuy, Pedro Pablo Gómez y Quimis. Table 1. List of plant species with medicinal interest for the inhabitants of Membrillal, Julcuy, Pedro Pablo Gómez and Quimis.

\begin{tabular}{clll}
\hline Número & \multicolumn{1}{c}{ Nombre vernáculo } & \multicolumn{1}{c}{ Nombre científico } & Familia \\
\hline 1 & achiote & Bixa orellana $L$ & Bixaceae \\
2 & achochilla & Momordica charantia $L$ & Cucurbitaceae \\
3 & aguacate & Persea americana Mill. & Lauraceae \\
4 & ajo & Allium sativum $L$. & Phytolaccaceae \\
5 & abacá & Musa textilis Née & Musaceae \\
6 & albahaca & Celosia virgata Jacq & Amaranthaceae \\
7 & albahaca & Ocimum campechianum Mill. & Lamiaceae \\
8 & albahaca de canela & Ocimum basilicum L & Lamiaceae \\
9 & alcanforero & Cinnamomum camphora (L.) J. Presl & Lauraceae \\
10 & alcohol de menta & Mentha piperita L. & Lamiaceae
\end{tabular}




\begin{tabular}{|c|c|c|c|}
\hline Número & Nombre vernáculo & Nombre científico & Familia \\
\hline 11 & alfalfa & Medicago sativa $L$. & Fabaceae \\
\hline 12 & algarrobo & Prosopis flexuosa DC & Fabaceae \\
\hline 13 & algarrobo & Prosopis pallida (Willd) Kunth & Fabaceae \\
\hline 14 & almendra & Terminalia catappa & Combretaceae \\
\hline 15 & almendro & Prunus dulcis (Mill.) D. A. Webb & Rosaceae \\
\hline 16 & altamisa & Artemisia vulgaris $L$. & Asteraceae \\
\hline 17 & altamisa & Tanacetum parthenium (L.) Sch. Bip. & Asteraceae \\
\hline 18 & angélica & Angelica archangelica $L$ & Apiaceae \\
\hline 19 & anís & Pimpinella anisum $L$. & Apiaceae \\
\hline 20 & anona & Annona reticulata $L$. & Annonaceae \\
\hline 21 & apio & Apium graveolens $L$ & Apiaceae \\
\hline 22 & arándano & Vaccinium myrtillus $L$. & Ericaceae \\
\hline 23 & árbol del pan & Artocarpus altilis (Parkinson) Fosberg & Moraceae \\
\hline 24 & badea & Passiflora quadrangularis $L$ & Passifloraceae \\
\hline 25 & bálsamo & Myroxylon peruiferum L.f. & Fabaceae \\
\hline 26 & bálsamo & Myroxylon balsamum (L.)Harms & Fabaceae \\
\hline 27 & barbosa & Barbarea vulgaris (L.) W.T. Aiton & Brassicaceae \\
\hline 28 & $\begin{array}{l}\text { bay rrun, pimienta de tabasco } \\
\text { o malagueta }\end{array}$ & Pimenta racemosa (Mill.) J. W. Moore & Myrtaceae \\
\hline 29 & bejuco bravo & Banisteriopsis caapi (Spruce ex Griseb.) Morton & Malpighiaceae \\
\hline 30 & bejuco estrella & Aristolochia trilobata L. & Aristolochiaceae \\
\hline 31 & berro & Nasturtium officinale W.T. Aiton & Brassicaceae \\
\hline 32 & bledo & Amaranthus Viridis & Amaranthaceae \\
\hline 33 & boldo & Peumus boldus Molina & Monimiaceae \\
\hline 34 & borraja & Borago officinalis $L$. & Boraginaceae \\
\hline 35 & buscapino o parietaria & Parietaria alsinifolia Del & Urticaceae \\
\hline 36 & coca & Erythroxylum coca Lam. & Malvaceae \\
\hline 37 & caimito & Pouteria caimito (Ruiz \& Pav.) Radlk & Sapotaceae \\
\hline 38 & canela & Cinnamomum verum J. S. Presl & Lauraceae \\
\hline 39 & caña agria & Costus spicatus (Jacq.) Sw. & Costaceae \\
\hline 40 & cascarilla & Cinchona pubescens Vahl & Rubiaceae \\
\hline 41 & cascarilla o kina & Cinchona officinalis $L$ & Rubiaceae \\
\hline 42 & cedrón & Aloysia citrodora Palau & Verbenaceae \\
\hline 43 & cerezo & Prunus cerasus $L$ & Rosaceae \\
\hline 44 & chácara & Cassia fistula $L$. & Fabaceae \\
\hline 45 & chaya & Cnidoscolus aconitifolius (Mill.) I.M.Johnst & Euphorbiaceae \\
\hline 46 & choclo morado & Zea mays $L$ & Poaceae \\
\hline 47 & chuchuhuasi & Monteverdia macrocarpa (Ruiz \& Pav.) Biral & Celastraceae \\
\hline 48 & cilantro & Coriandrum sativum $L$ & Apiaceae \\
\hline 49 & cilantro de pozo & Adiantum capillus-veneris $L$. & Pteridaceae \\
\hline 50 & ciruela & Prunus domestica L. & Rosaceae \\
\hline 51 & clavo de olor & Syzygium aromaticum (L.) Merr. \& Perry & Myrtaceae \\
\hline 52 & cojoba & Cojoba arborea (L.)Britton \& Rose & Fabaceae \\
\hline 53 & cojojo & Capsicum annuиm L. & Solanaceae \\
\hline 54 & congona & Peperomia inaequalifolia Ruiz \& Pav & Piperaceae \\
\hline 55 & culantro de pozo & Asplenium monanthes $L$ & Aspleniaceae \\
\hline 56 & curia & Justicia pectoralis Jacq & Acanthaceae \\
\hline 57 & dulcamara & Solanum dulcamara L. & Solanaceae \\
\hline
\end{tabular}




\begin{tabular}{|c|c|c|c|}
\hline Número & Nombre vernáculo & Nombre científico & Familia \\
\hline 58 & dulce limón & Citrus limetta Risso & Rutaceae \\
\hline 59 & espanto & Thunbergia alata Boj. ex Sims & Acanthaceae \\
\hline 60 & espino blanco & Crataegus monogyna Jacq. & Rosaceae \\
\hline 61 & eucalipto & Eucalyptus camaldulensis Dehnh. & Myrtaceae \\
\hline 62 & eucalipto & Eucalyptus globulus Labill. & Myrtaceae \\
\hline 63 & flor de loto & Nelumbo nucifera Gaertn. & Nelumbonaceae \\
\hline 64 & flor de muerto & Calendula officinalis $L$. & Asteraceae \\
\hline 65 & frijol de palo & Cajanus cajan (L.)Millsp & Fabaceae \\
\hline 66 & fruta del pan & Artocarpus altilis & Moraceae \\
\hline 67 & granado & Punica granatum $L$ & Lythraceae \\
\hline 68 & guanábana & Annona muricata $L$. & Annonaceae \\
\hline 69 & guayaba & Psidium guajava $L$ & Myrtaceae \\
\hline 70 & guineo morado & Musa acuminata Colla & Musaceae \\
\hline 71 & haba & Vicia faba $L$ & Fabaceae \\
\hline 72 & hieba de espanto & Thunbergia alata Boj. ex Sims & Acanthaceae \\
\hline 73 & hierba buena & Mentha spicata $L$. & Lamiaceae \\
\hline 74 & hierba luisa & Cymbopogon citratus (DC.) Stapf & Poaceae \\
\hline 75 & hierba mora & Solanum americanum subsp. americanum & Solanaceae \\
\hline 76 & hoja de aire & Kalanchoe pinnata (Lam.) Pers & Crassulaceae \\
\hline 77 & insulina & Chamaecostus cuspidatus (Nees \& Mart.) & Costaceae \\
\hline 78 & jengibre & Zingiber officinale Roscoe & Zingiberaceae \\
\hline 79 & johnson & Sorghum halepense (L.) Pers & Poaceae \\
\hline 80 & laurel & Cordia alliodora (Ruiz \& Pav.) Oken & Ehretiaceae \\
\hline 81 & limón & Citrus limon (L.) Burm. Fil & Rutaceae \\
\hline 82 & llantén & Plantago major L. & Plantaginaceae \\
\hline 83 & mango & Mangifera indica $L$. & Anacardiaceae \\
\hline 84 & manzanilla & Chamaemelum nobile (L.) All. & Asteraceae \\
\hline 85 & manzanilla de castilla & Matricaria chamomilla L. & Asteraceae \\
\hline 86 & marañón & Anacardium occidentale $L$ & Anacardiaceae \\
\hline 87 & mastrante & Lippia alba (Mill.) N.E.Br. ex Britton \& P.Wilson & Verbenaceae \\
\hline 88 & mastranto & Mentha suaveolens Ehrh & Lamiaceae \\
\hline 89 & matapalo & Ficus benghalensis L & Moraceae \\
\hline 90 & mate & Crescentia cujete L. & Bignoniaceae \\
\hline 91 & menta, meta japonesa & Mentha arvensis $L$. & Lamiaceae \\
\hline 92 & menta, toronjil de menta & Mentha piperita $L$ & Lamiaceae \\
\hline 93 & moringa & Moringa oleifera Lam. & Moringaceae \\
\hline 94 & muñeco & Cordia eriostigma Pittier & Ehretiaceae \\
\hline 95 & muyuyo & Cordia lutea Lam. & Boraginaceae \\
\hline 96 & naranja amarga & Citrus $\times$ aurantium $L$ & Rutaceae \\
\hline 97 & naranja agria & Citrus $\times$ aurantiifolia (Christm.) Swingle & Rutaceae \\
\hline 98 & naranjo & Citrus sinensis & Rutaceae \\
\hline 99 & neem & Azadirachta indica A. Juss. & Meliaceae \\
\hline 100 & noni & Morinda citrifolia L., nom. cons. & Rubiaceae \\
\hline 101 & nopal & Opuntia ficus-indica (L.) Mill. & Cactaceae \\
\hline 102 & oreganito & Lippia micromera Schauer & Verbenaceae \\
\hline 103 & orégano grande & Origanum vulgare $L$. & Lamiaceae \\
\hline 104 & oreganón & Plectranthus amboinicus (Lour.) Spreng. & Lamiaceae \\
\hline 105 & ortiga & Urtica dioica $L$ & Urticaceae \\
\hline
\end{tabular}




\begin{tabular}{|c|c|c|c|}
\hline Número & Nombre vernáculo & Nombre científico & Familia \\
\hline 106 & paico & $\begin{array}{l}\text { Dysphania ambrosioides (L.) Mosyakin \& } \\
\text { Clemants }\end{array}$ & Amaranthaceae \\
\hline 107 & palo santo & Bursera graveolens (Kunth) Triana \& Planch. & Burseaceae \\
\hline 108 & palomo & Geranium columbinum L. & Geraniáceas. \\
\hline 109 & pepino & Cucumis sativus L. & Cucurbitaceae \\
\hline 110 & pepito colorado & Erythrina velutina Willd. & Fabaceae \\
\hline 111 & perejil & Petroselinum crispum subsp. Crispum & Apiaceae \\
\hline 112 & periquito, perica & Mirabilis jalapa $L$. & Nyctaginaceae \\
\hline 113 & perlilla & Margyricarpus pinnatus (Lam.) Kuntze & Rosaceae \\
\hline 114 & perlillo & Vallesia glabra & Apocynaceae \\
\hline 115 & piña & Ananas comosus (L.) Merr. & Bromeliaceae \\
\hline 116 & piñón & Jatropha curcas $L$. & Euphorbiaceae \\
\hline 117 & plátano & Musa paradissiaca $L$. & Musaceae \\
\hline 118 & porcelana & Alpinia nutans (L.) Roscoe & Zingiberaceae \\
\hline 119 & porcelana & Begonia semperflorens Link y Otto & Begoniaceae \\
\hline 120 & $\begin{array}{l}\text { puta de noche (dama de } \\
\text { noche) }\end{array}$ & Cestrum nocturnum L. & Solanaceae \\
\hline 121 & romero & Rosmarinus officinalis $L$. & Lamiaceae \\
\hline 122 & rosa de muerto & Tagetes patula L. & Asteraceae \\
\hline 123 & ruda de castilla & Ruta graveolens $L$. & Rutaceae \\
\hline 124 & ruda de gallinaza & Porophyllum ruderale (Jacq.) Cass. & Asteraceae \\
\hline 125 & sábila & Aloe vera (L.) Burm.f. & Asphodelaceae \\
\hline 126 & sasafrás & Sassafras albidum (Nutt.) Nees & Lauraceae \\
\hline 127 & sauco negro & Sambucus nigra L. & Adoxaceae \\
\hline 128 & stevia & Stevia rebaudiana (Bertoni) Bertoni & Asteraceae \\
\hline 129 & tabaco & Nicotiana tabacum L. & Solanaceae \\
\hline 130 & tagua & Phytelephas aequatorialis Spruce & Arecaceae \\
\hline 131 & tamarindo & Tamarindus indica $L$. & Fabaceae \\
\hline 132 & té verde & Hygrophila serpyllum (Nees) T.Anderson & Acanthaceae \\
\hline 133 & teatina & Ageratum conyzoides $L$. & Asteraceae \\
\hline 134 & $\begin{array}{l}\text { teatino, escobilla, paraguay } \\
\text { y yerbaní }\end{array}$ & Scoparia dulcis L. & Plantaginaceae \\
\hline 135 & torinjil & Melissa officinalis $L$. & Lamiaceae \\
\hline 136 & toronja & Citrus grandis (L.) & Rutaceae \\
\hline 137 & valeriana & Valeriana acutiloba Rydb. & Caprifoliaceae \\
\hline 138 & valeriana & Valeriana officinalis $L$. & Caprifoliaceae \\
\hline 139 & verbena & Verbena officinalis $L$. & Verbenaceae \\
\hline 140 & verdolaga & Portulaca quadrifida L. & Portulacaceae \\
\hline 141 & yuca & Manihot esculenta Crantz & Euphorbiaceae \\
\hline 142 & $\begin{array}{l}\text { zapote, zapotillo de Perú, } \\
\text { chupa-chupa }\end{array}$ & Quararibea cordata (Bonpl.) Vischer & Malvaceae \\
\hline 143 & zaragosa & Aristolochia baetica L. & Aristolochiaceae \\
\hline 144 & zorrilla & Petiveria alliacea L. & Phytolaccaceae \\
\hline
\end{tabular}

En la Figura 1 se presentan los resultados de las partes de las plantas que se utilizan con diversos fines en las localidades de Membrillal, Julcuy, Pedro Pablo Gómez y Quimis. La forma de uso fue otra de las preguntas presentadas en la entrevista realizada en este estudio; en este sentido, los entrevistados mencionaron utilizar con mayor frecuencia la infusión y con menor frecuencia los emplastos. En lo que respecta al lugar donde obtienen las plantas medicinales, el $94 \%$ de los entrevistados mencionaron que de los patios o huertos caseros. En cuanto a la cantidad de veces que los 
entrevistados hacen uso de las plantas medicinales se confirmó que el 98,55 \% de dichas personas usan las plantas cuando lo necesitan, sin importar la época del año. Otro aspecto considerado en las entrevistas es la distancia (km) a la que se encuentran los entrevistados de las áreas en donde crecen las plantas de interés medicinal; en este sentido el 95,65\% hicieron referencia a que obtienen dichas plantas a distancias que oscilan entre 0 y $5 \mathrm{~km}$.

Figura 1. Partes de las plantas que se utilizan como medicina natural y tradicional por las localidades Membrillal, Julcuy, Pedro Pablo Gómez y Quimis.

Figure 1. Parts of the plants that are used as natural and traditional medicine in the Membrillal, Julcuy, Pedro Pablo Gómez and Quimis localities.

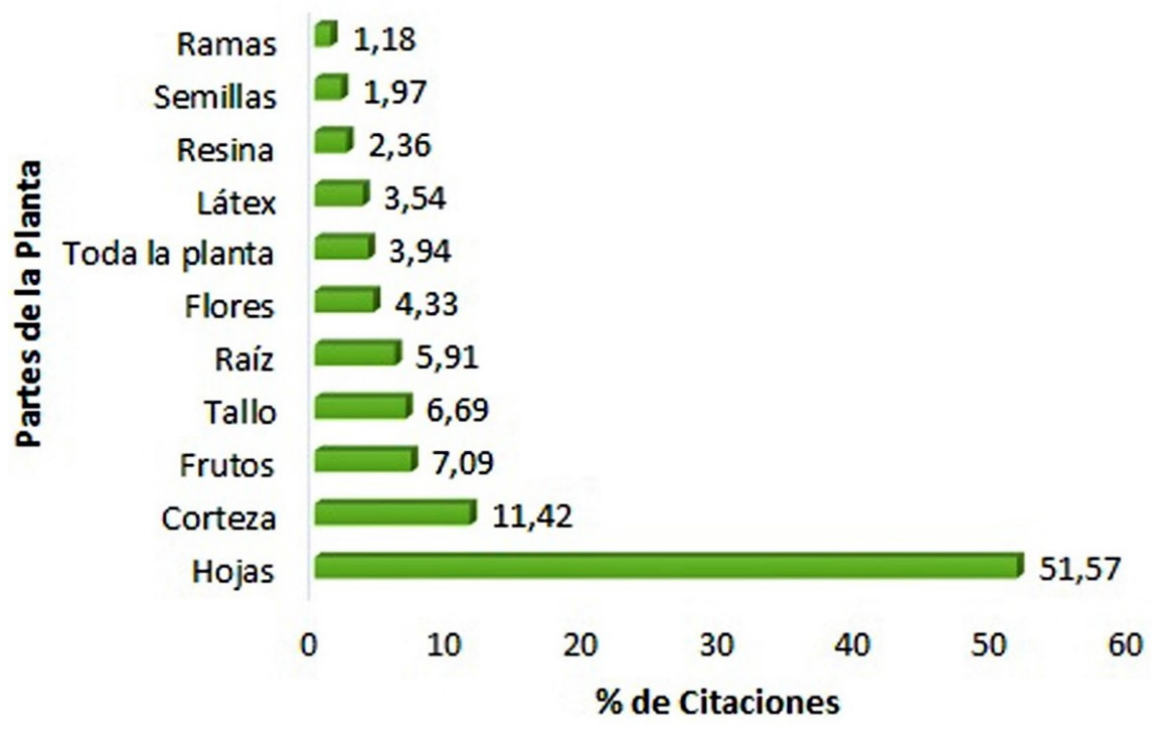

El uso más frecuente que los entrevistados les dan a las plantas de interés medicinal está enfocado al consumo y el uso menos frecuente es el comercio. En cuanto a la época de recolección, el 67,19 \% de los entrevistados recolectan las plantas medicinales todo el año. Otros aspectos indagados fueron las principales enfermedades y afecciones tratadas con plantas medicinales, según información proporcionada por los entrevistados (Tabla 2).

Tabla 2. Principales enfermedades que afectan a los entrevistados en las localidades de Membrillal, Quimis, Julcuy y Pedro Pablo Gómez. Table 2. Main diseases that regularly affect to the interviewees in the localities of Membrillal, Quimis, Julcuy and Pedro Pablo Gómez.

\begin{tabular}{lcccc}
\hline & Membrillal & Julcuy & Pedro Pablo Gómez & Quimis \\
\cline { 2 - 4 } Sistemas del Cuerpo Humano & \multicolumn{2}{c}{ \% de Uso } \\
\hline Sistema gastrointestinal & 35,94 & 27,87 & 30,30 & 35,00 \\
Sistema reproductivo & 6,00 & 8,00 & 5,00 & 5,00 \\
Sistema respiratorio & 9,60 & 16,00 & 14,70 & 15,00 \\
Sistema sensorial & 14,50 & 12,00 & 12,00 & 10,00 \\
Sistema osteomuscular & 10,76 & 11,00 & 11,00 & 10,00 \\
Sistema inmunológico & 7,20 & 6,80 & 7,00 & 7,00 \\
Sistema renal/urológico & 7,00 & 8,00 & 9,00 & 7,00 \\
Afecciones de la piel & 9,00 & 10,33 & 11,00 & 11,00 \\
\hline
\end{tabular}

La Tabla 3 muestra los resultados de las características fitoquímicas de las 15 especies de plantas de interés medicinal mencionadas con mayor frecuencia por los entrevistados en las localidades objeto de estudio. 
Tabla 3. Características fitoquímicas de las especies de interés medicinal en las localidades de Membrillal, Julcuy Pedro Pablo Gómez y Quimis.

Table 3. Phytochemical characteristics of the plant species with medicinal interest in the localities of Membrillal, Julcuy Pedro Pablo Gómez and Quimis.

\begin{tabular}{|c|c|c|c|c|c|c|c|c|c|c|}
\hline \multirow{2}{*}{ Especies } & \multicolumn{9}{|c|}{ Características fitoquímicas } & \multirow[b]{2}{*}{ Quinonas } \\
\hline & Alcaloides & Saponinas & Taninos & Fenoles & Cumarinas & Catequinas & Flavonoides & Triterpenos & Terpenoides & \\
\hline $\begin{array}{l}\text { Mentha } \\
\text { spicata }\end{array}$ & & & & & & & $\mathrm{X}$ & & & \\
\hline $\begin{array}{l}\text { Cymbopo- } \\
\text { gon citratus }\end{array}$ & $\mathrm{X}$ & $\mathrm{X}$ & & $\mathrm{X}$ & & & $X$ & & & \\
\hline $\begin{array}{l}\text { Origanum } \\
\text { vulgare }\end{array}$ & $\mathrm{X}$ & & & $\mathrm{X}$ & & & $X$ & $\mathrm{X}$ & $\mathrm{X}$ & \\
\hline $\begin{array}{l}\text { Ruta } \\
\text { graveolens }\end{array}$ & $\mathrm{X}$ & & & $\mathrm{X}$ & & & $\mathrm{X}$ & $\mathrm{X}$ & & \\
\hline Aloe vera & & $\mathrm{X}$ & & $\mathrm{X}$ & $\mathrm{X}$ & & $\mathrm{X}$ & $\mathrm{X}$ & & $\mathrm{X}$ \\
\hline $\begin{array}{l}\text { Thunbergia } \\
\text { alata }\end{array}$ & & & & & $\mathrm{X}$ & & $\mathrm{X}$ & & & \\
\hline $\begin{array}{l}\text { Mentha } \\
\text { piperita }\end{array}$ & $\mathrm{X}$ & & & & $\mathrm{X}$ & & & & $\mathrm{X}$ & \\
\hline $\begin{array}{l}\text { Lippia } \\
\text { micromera }\end{array}$ & $\mathrm{X}$ & $\mathrm{X}$ & $X$ & $\mathrm{X}$ & & $\mathrm{X}$ & $\mathrm{X}$ & $\mathrm{X}$ & & $\mathrm{X}$ \\
\hline $\begin{array}{l}\text { Matricaria } \\
\text { chamomilla }\end{array}$ & & & & $\mathrm{X}$ & $\mathrm{X}$ & & $\mathrm{X}$ & $\mathrm{X}$ & $\mathrm{X}$ & \\
\hline $\begin{array}{l}\text { Plectrantus } \\
\text { amboinicus }\end{array}$ & $\mathrm{X}$ & & & $\mathrm{X}$ & $\mathrm{X}$ & X & $\mathrm{X}$ & $\mathrm{X}$ & & $\mathrm{X}$ \\
\hline $\begin{array}{l}\text { Bursera } \\
\text { graveolens }\end{array}$ & & $\mathrm{X}$ & & $\mathrm{X}$ & & & $\mathrm{X}$ & $\mathrm{X}$ & & \\
\hline $\begin{array}{l}\text { Moringa } \\
\text { oleifera }\end{array}$ & $\mathrm{X}$ & & $\mathrm{X}$ & $\mathrm{X}$ & & & $\mathrm{X}$ & & & \\
\hline $\begin{array}{l}\text { Plantago } \\
\text { rugelii }\end{array}$ & $\mathrm{X}$ & $X$ & & $\mathrm{X}$ & X & & $\mathrm{X}$ & X & & \\
\hline $\begin{array}{l}\text { Eucalyptus } \\
\text { camaldu- } \\
\text { lensis }\end{array}$ & & & $\mathrm{X}$ & & & $\mathrm{X}$ & $\mathrm{X}$ & & & $\mathrm{X}$ \\
\hline $\begin{array}{l}\text { Psidium } \\
\text { guajava }\end{array}$ & & $\mathrm{X}$ & $\mathrm{X}$ & $\mathrm{X}$ & & & $\mathrm{X}$ & $\mathrm{X}$ & $\mathrm{X}$ & \\
\hline
\end{tabular}

\section{Discusión}

La investigación obtenida sobre las plantas de interés medicinal, indica que el mayor porcentaje de citas lo alcanzaron las especies Mentha spicata y Cymbopogon citratus, dato que coincide con otros estudios de uso de plantas medicinales, que mencionaron dichas especies como las más utilizadas por los habitantes de la comunidad serrana de Corralillo Arriba (Escalona Cruz et al., 2015).

Respecto a las categorías de enfermedades, los resultados obtenidos muestran que las categorías más mencionadas fueron "Otros síntomas" y "Enfermedades del sistema digestivo, infecciosas y parasitarias", estos resultados coinciden con la investigación de Jacob Paredes et al. (2015), quienes reportaron las afecciones o las enfermedades digestivas, parasitosis intestinal y enfermedades respiratorias, como las más tratadas con las plantas medicinales, aspecto que también coincide con los reportes de da Silva et al. (2017).

En el caso de los entrevistados en Membrillal (35,94 \%); Julcuy (27,87 \%); Pedro Pablo Gómez (30,3 \%) y Quimis $(35,0 \%)$, plantearon que utilizan las plantas con mayor frecuencia para curar problemas gastrointestinales. Estos resultados concuerdan con el estudio realizado por Valdés-Cobos (2013), en México, quienes reportaron enfermedades relacionadas con los problemas gastrointestinales y respiratorios, también el trabajo de Angulo Colimba et al. (2012), en Colombia, en el que las enfermedades más atendidas con plantas medicinales fueron las gastrointestinales; y también con la investigación de Jacob Paredes et al. (2015), en la provincia de Los Ríos en Ecuador. La desviación estándar para las enfermedades del sistema gastrointestinal $\left(\mathrm{S}^{2}=3,83\right)$ y el coeficiente de variación $(\mathrm{CV}=0,11)$. 
En relación con las partes de plantas más utilizadas por los entrevistados, las hojas (65,5\%), en particular en Quimis el $100 \%$ de las personas entrevistadas las usan para infusiones, así la desviación estándar $\left(\mathrm{S}^{2}=11,5\right)$ y el coeficiente de variación $(\mathrm{CV}=0,35)$ entre las cuatro localidades; le sigue en orden de usos la corteza y los tallos $(14,0$ y 14,5 \%, respectivamente), con $\left(S^{2}=8,87\right)$ y $(C V=1,27)$ para el uso de la corteza y de $\left(S^{2}=7,80\right)$ y $(C V=0,84)$ para el uso del tallo o parte de éste, entre localidades, respectivamente. Los valores del CV obtenidos en el caso de las hojas y los tallos pueden estar indicando el papel jugado por la familia en la trasmisión de saberes y prácticas ancestrales durante generaciones de pobladores establecidos por siglos en los diferentes sitios de la zona sur de Manabí.

Los resultados alcanzados en esta investigación corroboran los estudios etnobotánicos de da Silva et al. (2017), Zambrano-Intriago et al. (2015) y Jacob Paredes et al. (2015), quienes reportaron a las hojas como las más utilizadas, seguido de la raíz, la corteza, los tallos, las flores y los frutos, aspecto que puede indicar que en las localidades estudiadas se hace un uso más racional de las plantas de interés medicinal, ya que el uso de las partes aéreas (hojas, corteza, tallos) es más sostenible que usar otras partes de las plantas como las raíces o la planta completa, como lo mencionaron Jiménez González et al. (2017).

Otros autores como Manzanero-Medina et al. (2009) plantearon que el manejo de aquellas especies medicinales cuyos usos están en las raíces se realiza empezando por recolectar las raíces con el empleo de una pala o machete; para esos autores se toma solo la porción de la raíz que van a utilizar, mientras que la otra parte se queda en el suelo, lo que permite que las plantas tengan tiempo de recuperarse y reproducirse nuevamente mediante su semilla (una vez crezcan de nuevo a partir de esas partes que quedaron en el suelo, por ejemplo, mediante estolones) y en su mismo hábitat, luego las raíces se preparan dependiendo de la especie, por maceración alcohólica y mediante infusiones; de acuerdo con los resultados obtenidos en esta investigación, en Membrillal y Julcuy el $8 \%$ de los entrevistados expresaron que utilizan las raíces de las plantas para preparar algún producto medicinal, el resto de las localidades el $1 \%$ y el $3 \%$ para Pedro Pablo Gómez y Quimis, respectivamente.

De acuerdo con la información suministrada por las personas entrevistadas en las cuatro localidades, los métodos de elaboración o la forma de preparación más utilizada es la infusión (70,5\%) mientras que la forma menos utilizada es el emplasto ( $8 \%$ ); en este sentido los datos recolectados apuntan a que la mayoría de las personas entrevistadas hacen infusiones, principalmente con las hojas de las plantas, con porcentajes de 100, 98, 54 y 30 \%, para Pedro Pablo Gómez, Membrillal, Quimis y Julcuy, respectivamente; estos resultados coinciden con el estudio etnobotánico de Buitrago Zapata et al. (2018), quienes reportaron que en tres municipios de Antioquia el 61,1\% preparan infusiones a partir de las plantas medicinales y una de las formas menos comunes es el emplasto; así mismo, coinciden con Zambrano et al., (2015); los estadísticos desviación estándar $\left(\mathrm{S}^{2}=17,17\right)$ y el coeficiente de variación $(\mathrm{CV}=0,48)$, así lo demuestran.

El $71 \%$ de los entrevistados, es decir, el mayor porcentaje, mencionaron que obtienen las plantas de interés medicinal usadas por ellos de los huertos o patios caseros, en menor porcentaje (7\%) dijeron que hacen colectas en matorral con una desviación estándar $\left(S^{2}=9,81\right)$ y el coeficiente de variación $(\mathrm{CV}=0,27)$; estos resultados coinciden con lo planteado por Escalona Cruz et al. (2015), quienes reportaron que el $71 \%$ de las plantas informadas se cultivan en los patios de las casas.

En lo concerniente a las sustancias bioactivas que, según la literatura, contienen las plantas medicinales mencionadas por los entrevistados de Membrillal, Julcuy, Pedro Pablo Gómez y Quimis, se corrobora lo planteado por Carvajal Rojas et al. (2009); Ramírez Cárdenas et al. (2017) y Pérez-Chauca et al. (2020), quienes confirmaron la presencia de alcaloides, esteroides o triterpenoides libres, flavonoides, taninos, saponinas y lactonas sesquiterpénicas en plantas medicinales citadas en sus investigaciones.

El conocimiento de los principios bioactivos que poseen las personas sobre las plantas existentes en los ecosistemas de la zona sur de Manabí servirá de punto de partida para futuras investigaciones en la rama de la bioquímica. Este hecho, según Beyra et al. (2004); Ugartemendia-Ugalde et al. (2015), a su vez contrasta con el creciente interés del mundo académico por conocer los principios bioactivos de las especies consideradas como medicinales; esos autores desarrollaron investigación bibliográfica partiendo de las plantas catalogadas como medicinales, como por ejemplo, especies de los géneros: Mentha, Matricaria, Valeriana, Passiflora, Citrus, entre otros; por otra parte, Jaramillo-Jaramillo et al. (2016) plantearon que el contenido de metabolitos secundarios hallados en plantas les atribuye un gran valor farmacológico.

\section{Conclusiones}

Las plantas medicinales más importantes para las localidades Membrillal, Julcuy, Pedro Pablo Gómez y Quimis son Mentha spicata y Cymbopogon citratus y el uso principal es la infusión, realizada con las hojas. 
Las características fitoquímicas más presentes en las plantas de interés medicinal de las localidades de Membrillal, Julcuy, Pedro Pablo Gómez y Quimis son los flavonoides, fenoles, triterpenos y alcaloides.

\section{Agradecimientos}

Los autores agradecemos a las comunidades de Membrillal, Julcuy, Pedro Pablo Gómez y Quimis por el apoyo brindado para la realización de este proyecto y también a la Universidad Estatal del Sur de Manabí por el financiamiento.

\section{Financiamiento}

Este proyecto ha sido financiado por la Universidad Estatal del Sur de Manabí.

\section{Contribuciones de los autores}

- Alfredo Jiménez González: conceptualización, investigación, metodología, recursos, redacción - revisión y edición.

- Karla Julexi Mora Zamora: investigación, software, redacción - borrador original.

- Sonia Rosete Blandariz: validación, redacción - revisión y edición.

- César Alberto Cabrera Verdesoto: análisis formal, investigación, visualización.

\section{Referencias}

Aguirre, Z. (2012). Guía para estudiar los productos forestales no madereros (PFNM). Documento de trabajo para estudiantes de la carrera de Ingeniería Forestal. Universidad Nacional de Loja.

Angulo Colimba, A. F., Rosero Garcés, R. A., \& González Insuasti, M. S. (2012). Estudio etnobotánico de las plantas medicinales utilizadas por los habitantes del corregimiento de Genoy, Municipio de Pasto, Colombia. Universidad y Salud, 14(2), 168-185. https://revistas.udenar.edu.co/index.php/usalud/article/view/1277

Bermúdez, A., Oliveira-Miranda, M. A., \& Velázquez, D. (2005). La investigación etnobotánica sobre plantas medicinales: una revisión de sus objetivos y enfoques actuales. Interciencia, 30(8), 453-459. http:// ve.scielo.org/scielo.php?script=sci_arttext\&pid=S0378-18442005000800005\&lng=es\&tlng=es.

Beyra, Á., León, M. C., Iglesias, E., Ferrándiz, D., Herrera, R., Volpato, G., Godínez, D., Guimarais, M., \& Álvarez, R. (2004). Estudios etnobotánicos sobre plantas medicinales en la provincia de Camagüey (Cuba). Anales Del Jardín Botánico De Madrid, 61(2), 185-203. https://doi.org/10.3989/ajbm.2004.v61.i2.44

Buitrago Zapata, H., Palacios Palacios, G., Perea Moreno, L. P., \& Hincapié Llanos, C. A. (2018). Estudio etnobotánico de plantas medicinales en tres municipios de Antioquia, Colombia. Revista Cubana de Plantas Medicinales, 23(4). http://www.revplantasmedicinales.sld.cu/index.php/pla/article/view/665/348

Carvajal Rojas, L., Hata Uribe, Y., Sierra Martínez, N., \& Rueda Niño, D. (2009). Análisis fitoquímico preliminar de hojas, tallos y semillas de cupatá (Strychnos schultesiana Krukoff). Revista Colombia Forestal, 12(1), 161-170. http://www.scielo.org.co/scielo.php?script=sci_arttext\&pid=S0120-07392009000100011

da Silva, W. B., Cajaiba, R. L., \& Möller Parry, M. (2017). Levantamento etnobotânico de plantas medicinais utilizadas pelos moradores do município de Uruará, estado do Pará, Brasil. Revista Cubana de Plantas Medicinales, 22(4). http://www.revplantasmedicinales.sld.cu/index.php/pla/article/view/696

Escalona Cruz, L., Tase Aguilar, A., Estrada Martínez, A., \& Almaguer MojenaI, M. L. (2015) .Uso tradicional de plantas medicinales por el adulto mayor en la comunidad serrana de Corralillo Arriba. Guisa, Granma. Revista Cubana de Plantas Medicinales, 20(4). http://revplantasmedicinales.sld.cu/index.php/pla/article/view/274/146

Gallegos-Zurita, M. (2016). Las plantas medicinales: principal alternativa para el cuidado de la salud, en la población rural de Babahoyo, Ecuador. Anales de la Facultad de Medicina, 77(4), 327-332. http://www.scielo. org.pe/scielo.php?script=sci_arttext\&pid=S1025-55832016000400002\&lng=es\&nrm=iso\&tlng=es 
Gil-Rodríguez, J. R., Herrera-Rojas, M. F., Mitre-Velasco, Y., \& Santamaria-Rivas, C. (2020). Compuestos activos en plantas utilizadas en la medicina tradicional mexicana. Revista RD-ICUAP, 6(1), 175-200. http://www.apps.buap.mx/ojs3/index.php/rdicuap/article/view/1746

Jacob Paredes, D., Buenaño-Allauca, M. P., \& Mancera-Rodríguez, N. J. (2015). Usos de plantas medicinales en la comunidad San Jacinto del cantón Ventanas, Los Ríos-Ecuador. Revista U.D.C.A Actualidad \& Divulgación Cientifica, 18(1), 39-50. http://dx.doi.org/10.31910/rudca.v18.n1.2015.452

Jaramillo-Jaramillo, C., Jaramillo-Espinoza, A., D’Armas, H., Troccoli, L., \& Rojas de Astudillo, L. (2016). Concentraciones de alcaloides, glucósidos cianogénicos, polifenoles y saponinas en plantas medicinales seleccionadas en Ecuador y su relación con la toxicidad aguda contra Artemia salina. Revista de Biología Tropical, 64(3), 1171-1184. https://revistas.ucr.ac.cr/index.php/rbt/article/view/19537

Jiménez González, A., Pincay Alcivar, F., Ramos Rodríguez, M., Mero Jalca, O., \& Cabrera Verdesoto, C. (2017). Utilización de productos forestales no madereros por pobladores que conviven en el bosque seco tropical. Revista Cubana de Ciencias Forestales, 5(3), 270-286. https://cfores.upr.edu.cu/index.php/cfores/article/view/264

Jiménez González, A., Pionce Andrade, G. A., Sotolongo Sospedra, R., Ramos Rodríguez, M. (2016). Perturbaciones humanas sobre la composición y estructura del bosque semideciduo mesófilo, reserva de la biósfera Sierra del Rosario, Cuba. SATHIRI, 1(10), 196-206. https://doi.org/10.32645/13906925.185

Jiménez González, A., Rosete Blandariz, S., Cantos Cevallos, C. G., Tapia Zúñiga, M. V., Castro Ponce, S. I., Gras Rodríguez, R., \& Cabrera Verdesoto, C. A. (2021). Componentes de la diversidad biológica empleados por las familias manabitas en la medicina natural y tradicional ( $1^{\text {a }}$ ed.). MAWIL. https://doi. org/10.26820/978-9942-826-71-8

Jiménez-González, A., García-López, M. R., Sotolongo-Sospedra, R., González-González, M., \& Martínez-Olivia, M. (2010). Productos forestales no madereros en la comunidad Soroa, Sierra del Rosario. Revista Forestal Baracoa, 29(2), 83-88. http://www.actaf.co.cu/revista-forestal-baracoa.html

Kvist, L. P., Oré-Balbín, I. C., Gonzales, A., \& Llapapasca-Samaniego, D. C. (2001). Estudio de plantas medicinales en la Amazonía peruana: una evaluación de ocho métodos etnobotánicos. Folia Amazónica, 12(1-2), 53-73. https://doi.org/10.24841/fa.v12i1-2.305

López-Roldán, P., \& Fachelli, S. (2015). Metodología de la investigación social cuantitativa (1 $1^{\mathrm{a}}$ ed.). Universitat Autònoma de Barcelona.

Manzanero-Medina, G. I., Flores-Martínez, A., Sandoval-Zapotitla, E., \& Bye-Boettler, R. (2009). Etnobotánica de siete raíces medicinales en el mercado de Sonora de la ciudad de México. Polibotánica,1(27), 191-228. https://www.polibotanica.mx/ojs/index.php/polibotanica/article/view/796

Olascuaga-Castillo, K., Rubio-Guevara, S., Valdiviezo-Campos, J. E., \& Blanco-Olano, C. (2020). Desmodium molliculum (Kunth) DC (Fabaceae); Perfil etnobotánico, fitoquímico y farmacológico de una planta andina peruana. Ethnobotany Research and Applications, 19, 19. https://ethnobotanyjournal.org/index. php/era/article/view/1811

Organización de las Naciones Unidas para la Alimentación y la Agricultura [FAO] \& Programa de las Naciones Unidas para el Medio Ambiente [PNUMA]. (2020). El estado de los bosques del mundo 2020. Los bosques, la biodiversidad y las personas. FAO \& PNUMA. https://doi.org/10.4060/ca8642es

Organización Mundial de la Salud [OMS]. (2018). Clasificación Internacional de Enfermedades ICD-11. https://icd.who.int/es

Organización Panamericana de la Salud [20Informe de reunión del grupo de expertos en plantas medicinales. OPS. https://iris.paho.org/handle/10665.2/50479

Pérez-Chauca, E., Saldaña-Bobadilla, V., \& Minchan-Herrera, P. (2020). Etnobotánica, farmacología, fitoquímica y usos medicinales de huamanpinta en el Perú - Chuquiraga spinosa Less. (Asteraceae). Ethnobotany Research and Applications, 19, 22. https://ethnobotanyjournal.org/index.php/era/article/view/1831

Ramírez Cárdenas, A., Isaza Mejía, G., Pérez Cárdenas, J. E., Martínez Garzón, M. M. (2017). Estudio fitoquímico preliminar y evaluación de la actividad antibacteriana del Solanum dolichosepalum Bitter (frutillo). Revista Cubana de Plantas Medicinales, 22(1), 1-11. https://www.medigraphic.com/cgi-bin/new/ resumen.cgi?IDARTICULO $=76601$

Reyes Vargas, M. V., López León Ging, C. P., \& Ortega Ocaña, Á. F. (2019). Aprovechamiento de recursos naturales y culturales con fines turísticos. Caso de estudio comuna San Jacinto del Pindo, en la provincia de Pastaza (Ecuador). Siembra, 6(1), 039-049. https://doi.org/10.29166/siembra.v6i1.1721 
Rodríguez Guerra, Y., Valdés Sáenz, M., Hernández Ramos, H., \& Soria Re, S. (2019). Guía metodológica para la obtención de información en el estudio etnobotánico de especies forestales en comunidades amazónicas. Revista Cubana de Ciencias Forestales, 7(1), 98-110. https://cfores.upr.edu.cu/index.php/cfores/ article/view/368

Roskov, Y., Ower, G., Orrell, T., Nicolson, D., Bailly, N., Kirk, P. M., Bourgoin, T., DeWalt, R. E., Decock, W., Nieukerken, E. van, Zarucchi, J., \& Penev L. (eds). (2019). Species 2000 \& ITIS Catalogue of Life, 2019 Annual Checklist. Species 2000: Naturalis. http://www.catalogueoflife.org/annual-checklist/2019/

Torres, M., Paz, K., \& Salazar, F. G. (2006). Tamaño de una muestra para una investigación de mercado. Boletín electrónico, 02. Universidad Rafael Landívar. http://moodlelandivar.url.edu.gt/url/oa/fi/ProbabilidadEstadistica/URL_02_BAS02\%20DETERMINACION\%20TAMA\%C3\%91O\%20MUESTRA.pdf

Tropicos.org. (2020). Missouri Botanical Garden. https://tropicos.org

Ugartemendia-Ugalde, L., Bravo-Santos, R., Tormo-Molina, R., Cubero-Juànez, J., Rodrigo-Moratinos, A. B., \& Barriga-Ibars, C. (2015). Especies vegetales presentes en la comunidad del País Vasco con principios bioactivos beneficiosos sobre el ritmo del sueño. Medicina naturista, 9(1), 49-60. http://www.medicinanaturista.org/images/revistas/mn9\%281\%29.pdf

Valdés-Cobos, A. (2013). Conservación y uso de plantas medicinales: el caso de la región de la Mixteca Alta Oaxaqueña, México. Ambiente y Desarrollo, 17(33), 87-99. https://revistas.javeriana.edu.co/index.php/ ambienteydesarrollo/article/view/7044

Zambrano-Intriago, L. F., Buenaño-Allauca, M. P., Mancera-Rodríguez, N. J., \& Jiménez-Romero, E. (2015). Estudio etnobotánico de plantas medicinales utilizadas por los habitantes del área rural de la parroquia San Carlos, Quevedo, Ecuador. Universidad y Salud, 17(1), 97-111. https://revistas.udenar.edu.co/index. $\mathrm{php} / \mathrm{usalud} /$ article/view/2400 